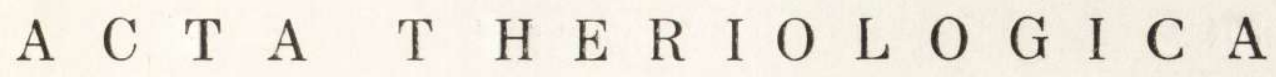

VOL. $20,24: 303-318$.

August, 1975

Clive D. JORGENSEN, H. Duane SMITH \& Del T. SCOTT

\section{Small Mammal Estimates Using Recapture Methods, with Variables Partitioned $^{1}$}

[With 5 Tables \& 1 Fig.

\begin{abstract}
Modifications and implementation of the model presented by $\mathrm{S} \mathrm{mith}$, Jorgensen \& Tolley (1972) $(E M-1)$ to estimate small mammal numbers and densities are described, along with some of the limitations. These modifications provide a practical model $(E M-2)$ that can be used to estimate small mammal populations, while at the same time, partitioning the estimator variables. Estimates can be obtained with confidence statements without dense-line data, or for that matter, without a dense-line even included in the field design.
\end{abstract}

\section{INTRODUCTION}

Historically, population ecologists have been concerned with estimating population parameters, such as distribution, population size, activity range, birth rate, death rate, age structure, density, biomass, etc. Before most of the parameters can be estimated, the number of organisms within a given area must be estimated by one of several possible methods that have been specifically designed to retain the population structure in its existing environment (P etersen, 1896; L incoln, 1930; Schnabel, 1938; Hayne, 1949; Leslie \& Chitty, 1951; Leslic, 195z; Jolly, 1963, 1965, and Smith, Jorgensen \& Tolley, 1972). Although all of these proposed methods were designed to satisfy specific sampling problems, the method described by $\mathrm{S} \mathrm{mith} \mathrm{et} \mathrm{al.,} 1972$ (hereafter referred to as $E M-1$ ) provided the most complete partitioning of the variables that are inevitably confounded in estimates of population size. This paper provides the basis for implementing $E M-1$ and is referred to as $E M-2$.

The field design and logic for the analytical method used to partition

1 This study was supported in part, by the US-IBP Desert Biome studies, contracted from Utah State University with Brigham Young University. 
the variables confounding estimates are explained by $\mathrm{Smith}$ et al. (1972). The logic is clear and EM-1 should be expected to provide reliable estimates of small mammal densities, if all of the assumptions are satisfied. Since the assumption of animal movement is not always satisfied and the general methods proposed are so restricted by this and other assumptions, a more robust model was required before the estimator could be very useful. Although fewer assumptions were required and EM-2 could be more widely applied, it sacrificed some accuracy when the assumptions of $E M-1$ were not completely satisfied.

This revised model (EM-2), with its redefinitions, is designed to provide estimates of mortality, trap avoidance, dispersal and population density when the dense-line yields no information about movement and/or the probability of trap avoidance is high from day $j$ to day $t$. Also, it provides an estimate of the population size $\left(N_{i}\right)$, with the above variables partitioned when a dense-line is not even included in the field design (Fig. 1).

\section{ANALYTICAL METHODS}

Perhaps the most elusive parameters effecting the response of esti-mators are the animal-trap relationships. Among these relationships is trap avoidance $\left(\hat{P a}_{i}\right)$, which is stated as the probability of a marked animal being recaptured exactly $i$ days after it was first caught, and estimated with:

$$
\hat{P a_{i}}=\left[\sum_{j=1}^{t-i} \frac{A_{j, j+1}}{n_{j}}\right] /{ }^{(t-i)}
$$

where: $A_{i j}=$ the $A_{i j}$ matrix described by J o lly $(1963,1965)$, and

$n_{j}=$ the number of animals caught on day $i$, that is the sample size on day $i$.

This estimate differs from a comparable estimate in EM-1 $\left(a_{i}\right)$, but its use is even more essential since it controls how the marked animals on the grid, that were never recaptured, are partitioned before $N_{i}$ is determined.

After $\hat{P a_{i}}$ has been determined, the probality that an animal left the grid after being captured can be estimated with:

$$
\hat{P} l_{i}=\left[\sum_{j=1}^{t-i} \frac{l t_{i},{ }_{j+1}}{n_{j}}\right] /{ }_{(t-i)}
$$

where: $l t_{i j}=$ a matrix containing the capture history of animals that left: the grid. The $l t_{i j}$ element is the number of grid residents that were last 
caught on the grid on day $i$, then caught on the dense-line (if present) on day $j$, but subsequently disappeared.

The probability of death $\left(\hat{P}_{i}\right)$ can now be determined with:

$$
\hat{P} d_{i+1}=1-\sum_{j=1}^{i}\left(\hat{P}_{t-j}+\hat{P} a_{t-j}\right)
$$

This probability represents the likelihood that an animal caught on day $i$, and never observed again, is dead, providing that $P d_{1}=1$. The number of animals that are not caught and assumed to be dead between day $i$ and day $t-1$ can be estimated with $d_{i}=\left(g_{i}\right)\left(\hat{P} d_{i+1}\right)$. In this case, $g_{i}$ is defined as the number of marked animals caught on day $i$ that were not caught during the rest of the trapping period.

$$
g_{i}=n_{i}-\sum_{j=i+1}^{t} A_{i j}
$$

where: $g_{i}=n_{i}$ less the number of grid residents caught on the dense-line when present) on the last trapping day, since grid residents may be caught on the dense-line on the last day.

Since it is not possible to determine precisely which days the $d_{i}$ animals died on, it is necessary to estimate the number of marked animals assumed to be dead from day 1 to day $t-1$ with:

$$
\hat{M} D_{i}=\sum_{j=1}^{i}\left(d_{i}\right)\left(\hat{P}_{t}-i+j\right)
$$

Now that the number of dead animals $\left(M D_{i}\right)$ can be estimated for day $i$, it is possible to estimate the number of marked animals on the grid at day $i$ with:

$$
\hat{M}_{i}=\sum_{j=1}^{i}\left(n_{j}+k_{j}-m_{j}-l_{j}-r_{j}\right)-\hat{M} D_{i}
$$

where: $k_{j}=$ the number of animals that immigrated to the grid and took up residency,

$m_{j}=$ the number of marked animals caught alive in the sample on day $i$,

$l_{j}=$ the number of marked animals that left the grid on day $i$, and

$r_{j}=$ the number of animals that died in the traps on day $i$. 
The number of animals moving onto the grid $\left(k_{i}\right)$ and the number of marked animals that move off the grid $\left(l_{i}\right)$ are determined with:

$$
\begin{aligned}
& k_{i}=\sum_{j=1}^{i} k t_{i j} \\
& l_{i}=\sum_{j=1}^{i} l t_{i j}
\end{aligned}
$$

where: $k t_{i j}=$ a matrix cantaining the capture history of animals that came onto the grid. The $k t_{i j}$ element is the number of animals that were last caught on the dense line on day $i$ that were subsequently caught on the grid on day $j$ then caught at least once more on the grid, but then never again caught on the dense-line.

The probability of capture $\left(\hat{\Phi}_{i}\right)$ for marked animals can be estimated with $\left(m_{i+1}+k_{i+1}\right) / \hat{M}_{i}$; thus, the population size $\left(N_{i}\right)$ on the grid can be estimated with:

$$
E M-2_{i}=\frac{\left(n_{i+1}+k_{i+1}\right)}{\left(m_{i+1}+k_{i+1}\right) / \hat{M}_{i}}
$$

Changes in the population during the sampling (trapping) period may now be expressed as $\Delta N_{i}=k_{i}+b_{i}-d_{i}-l_{i}$, where $b_{i}$ is the number born on day $i$ plus some random fluctuations due to errors in estimating $\Phi_{i}$. This might also be expressed as $\Delta N_{i}=\hat{N}_{i}-\hat{N}_{i}$, where $\hat{N}_{i}=E M-2_{i}$. The density of animals is then determined by taking the area contained within the grid and dividing it by $\hat{N}_{i}$.

As S mith et al. (1972) stated, estimates of population numbers should be accompanied by an appropriate confidence statement. The statement provided by them has been adopted as being the best, when modified such that $k_{j}=1$ when the dense-line is absent or when animals have not been detected to move onto the grid. Confidence for these estimates may then be obtained with:

$$
p\left\lfloor\frac{\hat{N}_{i}}{\hat{\Phi}_{i}+\boldsymbol{Z}_{\alpha / 2} \sqrt{\frac{\hat{\Phi}_{i}(1-\hat{\Phi})}{k_{i-1}}}}\right\rceil<\hat{N}_{i}<\left\lceil\frac{\hat{N}_{i}}{\hat{\Phi}_{i}-Z_{\alpha / 2}} \sqrt{\frac{\hat{\Phi}_{i}(1-\hat{\Phi})}{k_{i+1}}}\right]=1-\alpha
$$

III. METHODS

Field design for the grid and its accompanying dense-line are presented in Fig. 1. A more complete description of this design is provided by 
$\mathrm{S} \mathrm{mith} \mathrm{et} \mathrm{al.} \mathrm{(1972),} \mathrm{but} \mathrm{a} \mathrm{brief} \mathrm{illustration} \mathrm{here} \mathrm{is} \mathrm{useful} \mathrm{in} \mathrm{explaining}$ some of the experiments conducted to validate the estimator. Basically, data were gathered from a small mammal simulator where the variables could be controlled (Jorgensen, S mith \& S cott, 1972). The estimator (equation 9) will be illustrated with a set of data obtained from the small mammal simulator described by Jorgensen, Scott \& S mith (1972).

\section{RESULTS}

Data from the simulator included in Table 1 were converted to an $A_{i j}$ matrix (Table 2) for all animals caught on the grid. All other calculations

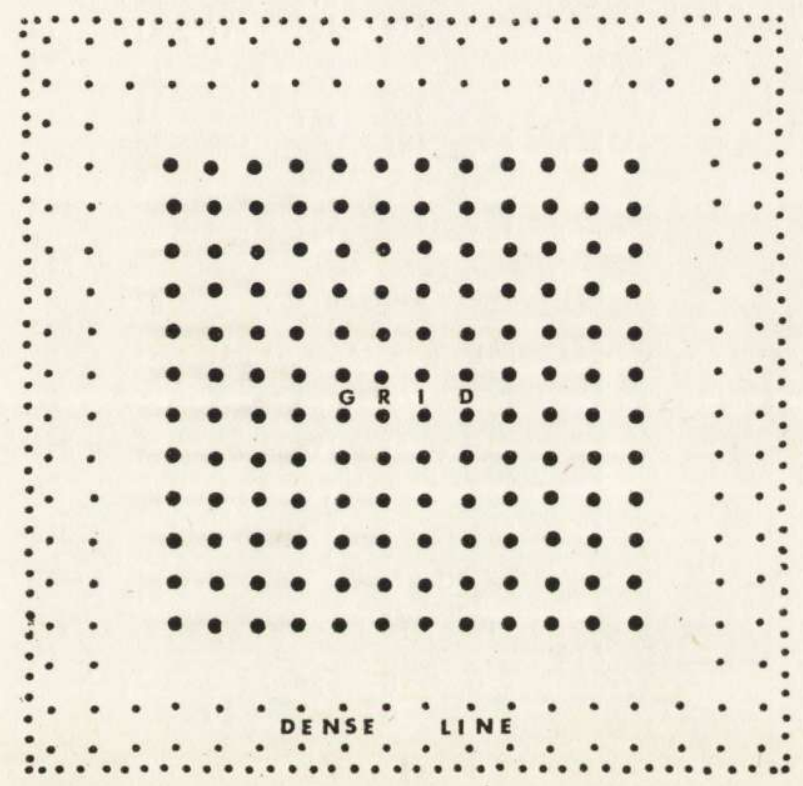

Fig. 1. Field design for the grid and its accompanying dense-line

are generated from this matrix, along with similar matrices for leaving the grid $\left(l t_{i j}\right)$ and coming onto the grid $\left(k t_{i j}\right)$. In this particular example animals did not move onto or from the grid; thus, the $\left(l t_{i j}\right)$ and $\left(k t_{i_{j}}\right)$ matrices were all zeros and provided no input into $\hat{M}_{i}$ (equation 6). Also, since the data were obtained from the simulator where population parameters could be controlled, the population was maintained stable at 49 .

Results of this example are reported in Table 3. If one is interested in a single estimate for the 10 day trapping period, there are several strategies that might be used. First, is simply a matter of using the mean (54.2) which is questionable because of the dependence of each estimate on those preceeding it. Secondly, a median (54) might also be used. This 
Table 1

Capture data of one class, trap numbers greater than 144 are dense-line traps.

\begin{tabular}{|c|c|c|c|c|c|c|c|c|c|c|c|}
\hline \multirow{2}{*}{$\begin{array}{l}\text { Animal } \\
\text { number }\end{array}$} & \multirow{2}{*}{$\begin{array}{l}\text { Percent } \\
\text { dense-line } \\
\text { captures }\end{array}$} & \multicolumn{10}{|c|}{ Days caught with station number } \\
\hline & & 1 & 2 & 3 & 4 & 5 & 6 & 7 & 8 & 9 & 10 \\
\hline 1 & 1.00 & & & & & & $168^{a}$ & & & 300 & \\
\hline 2 & 1.00 & & & & & 179 & 174 & 176 & 305 & 306 & \\
\hline 3 & 1.00 & $\therefore$ & & & & & & & & 165 & \\
\hline 4 & 1.00 & & & 153 & & & & & & 360 & \\
\hline 5 & 1.00 & 158 & 295 & 158 & 158 & & & & 161 & 159 & 159 \\
\hline 6 & 1.00 & & & 148 & 182 & 147 & & 289 & & 146 & 145 \\
\hline 7 & 1.00 & & 148 & 148 & & 148 & 290 & 184 & & 148 & 182 \\
\hline 8 & 1.00 & 181 & & & 179 & & 306 & & 181 & 183 & \\
\hline 9 & 1.00 & & & 178 & 183 & 177 & 177 & 175 & 179 & 176 & 178 \\
\hline 10 & 1.00 & & & & & & & 300 & 167 & 167 & \\
\hline 11 & 1.00 & 170 & & 166 & 168 & 170 & 301 & & & 166 & \\
\hline 12 & 1.00 & & & 169 & 167 & & 168 & & 168 & 168 & \\
\hline 13 & 1.00 & 297 & 298 & 161 & & 163 & 162 & & 297 & 162 & \\
\hline 14 & 1.00 & & & & 301 & & 169 & 170 & & 171 & 169 \\
\hline 15 & 1.00 & & 183 & 183 & 183 & 183 & & 183 & 180 & & \\
\hline 16 & 1.00 & 289 & 182 & 289 & 145 & 289 & 289 & & & 363 & 289 \\
\hline 17 & 1.00 & & & & & & & 296 & & & 296 \\
\hline 18 & 1.00 & 371 & & 175 & 176 & & 304 & & 303 & 304 & 371 \\
\hline 19 & 1.00 & & & & 305 & & & 305 & 177 & 177 & \\
\hline 20 & 1.00 & 294 & 361 & & & 361 & & & 293 & & \\
\hline 21 & 1.00 & 366 & 298 & 299 & 299 & 299 & 366 & & 366 & & \\
\hline 22 & 1.00 & 185 & & & 185 & 306 & 185 & & 187 & 187 & 183 \\
\hline 23 & 1.00 & & & & 173 & & 368 & 301 & 301 & & 368 \\
\hline 24 & 1.00 & 365 & 364 & & & & & & & 365 & 365 \\
\hline 25 & 1.00 & & & & & 370 & 369 & & 369 & & \\
\hline 26 & 1.00 & 152 & 359 & & 358 & & & 359 & & & \\
\hline 27 & 1.00 & & & & & & & & 364 & & \\
\hline 28 & 1.00 & & & & & & 370 & & & & \\
\hline 29 & 1.00 & & 370 & 374 & & & & & & & \\
\hline 30 & .67 & 374 & & & & & & 12 & & 374 & \\
\hline 31 & .00 & & 8 & 8 & 20 & & 7 & & 8 & & 8 \\
\hline 32 & .00 & & & 6 & 7 & 18 & & & & 18 & 6 \\
\hline 33 & 1.00 & & & & & & & & 311 & & \\
\hline 34 & .00 & & 6 & 18 & 5 & & & & 6 & & 18 \\
\hline 35 & .00 & & & & 13 & & & 2 & 13 & & \\
\hline 36 & .00 & 9 & & 22 & & 9 & 21 & & & 22 & 21 \\
\hline 37 & 1.00 & & & 313 & & & 198 & & 196 & 196 & 200 \\
\hline 38 & .00 & & 13 & & & 13 & & & & & \\
\hline $3 \theta$ & .00 & 20 & 20 & & 20 & 20 & & & & 19 & 21 \\
\hline 40 & 1.00 & 278 & & & & & & 378 & 314 & & 376 \\
\hline 41 & 1.00 & & 201 & & 376 & 380 & & 378 & 380 & & 314 \\
\hline 42 & .00 & 17 & & 30 & & & 15 & 29 & 17 & & \\
\hline 43 & .00 & & & & 21 & & 21 & & & & \\
\hline 44 & .00 & & & & & & 23 & 11 & & 36 & \\
\hline 45 & .00 & 29 & & & 17 & 29 & & 30 & & 17 & \\
\hline 46 & .00 & 15 & & 27 & & & & 26 & & 26 & \\
\hline 47 & .00 & & & & & 46 & 47 & 34 & & 33 & \\
\hline 48 & .00 & & & 28 & & 39 & & & & & \\
\hline 49 & .00 & 31 & 43 & 43 & & 43 & & 43 & 30 & & \\
\hline 50 & 1.00 & 202 & 206 & 315 & 319 & 206 & & & & & \\
\hline 51 & 1.00 & 382 & 382 & 211 & & & 382 & & 382 & 318 & 384 \\
\hline 52 & .00 & & 53 & & 42 & & & & & 42 & 41 \\
\hline 53 & 1.00 & & & & 212 & 208 & & 208 & & & \\
\hline 54 & .00 & 35 & & 48 & 48 & 60 & & & & 47 & 48 \\
\hline
\end{tabular}


Table 1, continued

\begin{tabular}{|c|c|c|c|c|c|c|c|c|c|c|c|}
\hline \multirow{2}{*}{$\begin{array}{l}\text { Animal } \\
\text { number }\end{array}$} & \multirow{2}{*}{$\begin{array}{l}\text { Percent } \\
\text { dense-line } \\
\text { captures }\end{array}$} & \multicolumn{10}{|c|}{ Days caught with station number } \\
\hline & & 1 & 、 2 & 3 & 4 & 5 & 6 & 7 & 8 & 9 & 10 \\
\hline 55 & 1.00 & & 320 & & & & & & & 320 & \\
\hline 56 & .00 & 55 & & 55 & & 55 & 42 & & $5 \overline{5}$ & & 56 \\
\hline 57 & 1.00 & & & & 215 & & & & & & \\
\hline 58 & .00 & 62 & 61 & 50 & & & & 52 & & & \\
\hline 59 & .20 & & 44 & & & 56 & 56 & & 68 & & 416 \\
\hline 60 & .00 & 59 & & & & & & & 59 & 59 & \\
\hline 61 & .00 & & 56 & 69 & & & & 69 & 69 & & \\
\hline 62 & .00 & & & 67 & 55 & 66 & & & & & 54 \\
\hline 63 & .00 & 62 & 62 & & & 61 & 61 & 61 & & 73 & \\
\hline 64 & .00 & 67 & & & & & & 67 & & & \\
\hline 65 & .00 & 72 & & 72 & & & & 84 & & & \\
\hline 66 & 1.00 & 322 & 386 & & 388 & & & & & 386 & \\
\hline 67 & .00 & 93 & & 56 & & 81 & & 81 & & & 69 \\
\hline 68 & .00 & 75 & 76 & & 83 & 76 & & 76 & & & \\
\hline 69 & .00 & & & & & 83 & 83 & 70 & 83 & & 83 \\
\hline 70 & 1.00 & 220 & & 220 & 218 & 222 & 218 & 220 & & 214 & 218 \\
\hline 71 & .00 & & & & 96 & & & & 84 & & \\
\hline 72 & .00 & & 81 & 81 & 69 & & & & & 70 & \\
\hline 73 & .00 & & & 91 & & & & & 80 & & \\
\hline 74 & .00 & & 79 & 79 & & 78 & 66 & & & & 79 \\
\hline 75 & .50 & & & 390 & & & & & & 84 & \\
\hline 76 & 1.00 & & & 325 & & & 222 & & & & 325 \\
\hline 77 & 1.00 & & & 221 & 221 & 221 & 219 & 221 & & & \\
\hline 78 & 1.00 & & & 389 & & & 389 & & 387 & 389 & 325 \\
\hline 79 & .00 & & 94 & 82 & 95 & & 95 & & 81 & & 95 \\
\hline 80 & .00 & & & 86 & & & & & & 85 & \\
\hline 81 & .00 & 88 & 88 & & & 88 & & & 88 & 88 & 89 \\
\hline 82 & 1.00 & 222 & 220 & & & & & & & & \\
\hline 83 & .00 & 106 & & & & 96 & & & & & \\
\hline 84 & .00 & & & & & & 108 & & . & & 96 \\
\hline 85 & .00 & 96 & 95 & 95 & & & 95 & & 107 & & \\
\hline 86 & .00 & & & & 92 & & & 105 & & 117 & \\
\hline 87 & .00 & 106 & 106 & 106 & & & 106 & 106 & & & 104 \\
\hline 88 & .00 & & & & & & 119 & & & & \\
\hline 89 & .00 & & & 107 & 106 & 118 & & 118 & & & 106 \\
\hline 90 & .00 & & & & & 97 & & 109 & & & \\
\hline 91 & 1.00 & & 328 & & & & & & & & \\
\hline 92 & .00 & & & & & & & & 101 & & \\
\hline 93 & 1.00 & & & & & & & & 395 & & \\
\hline 94 & .00 & & & & & & & & 116 & 116 & 116 \\
\hline 95 & 1.00 & 329 & & 327 & & & 395 & & 393 & & \\
\hline 96 & .00 & 117 & 129 & 116 & 128 & & & 117 & & 116 & 128 \\
\hline 97 & .00 & & & & 117 & 140 & & & 130 & & \\
\hline 98 & .00 & & & 128 & & & & 128 & 129 & & \\
\hline 99 & .00 & 144 & 131 & 131 & & 119 & 131 & 130 & & & 131 \\
\hline 100 & 1.00 & & & & & 334 & 332 & & & & \\
\hline 201 & 1.00 & & & 331 & & & & & & 395 & \\
\hline 102 & .00 & & & 129 & & & 129 & & 129 & 129 & 129 \\
\hline 03 & .00 & & & 125 & 136 & & 137 & 138 & 125 & & \\
\hline 104 & 1.00 & & & & & 395 & 395 & & & 395 & \\
\hline 305 & 1.00 & 241 & & & & & & & & & \\
\hline 306 & .00 & & 129 & & 129 & & & 139 & 140 & & \\
\hline 207 & .00 & & & 121 & & & & 133 & 133 & & \\
\hline 108 & .00 & & 135 & & & & & 136 & & 135 & \\
\hline 109 & 1.00 & 334 & 398 & 394 & 334 & & 398 & & 398 & & \\
\hline
\end{tabular}


Table 1 , continued

\begin{tabular}{|c|c|c|c|c|c|c|c|c|c|c|c|}
\hline \multirow{2}{*}{$\begin{array}{l}\text { Animal } \\
\text { number }\end{array}$} & \multirow{2}{*}{$\begin{array}{l}\text { Percent } \\
\text { dense-line } \\
\text { captures }\end{array}$} & \multicolumn{10}{|c|}{ Days caught with station number } \\
\hline & & 1 & 2 & 3 & 4 & 5 & 6 & 7 & 8 & 9 & 10 \\
\hline 110 & 1.00 & 333 & 238 & & 333 & & & 238 & & & 238 \\
\hline 111 & .00 & & 142 & & & & 130 & & & & \\
\hline 112 & .00 & & 141 & & 129 & & & & & 142 & \\
\hline 113 & 1.00 & 238 & 242 & & & 248 & & & & & 335 \\
\hline 114 & 1.00 & & 239 & & & & & & & & 241 \\
\hline 115 & .50 & & & 136 & & & 407 & & & & \\
\hline 116 & 1.00 & & & & & & 400 & & & 400 & \\
\hline 117 & 1.00 & & & & & & 414 & 415 & & & 414 \\
\hline 118 & 1.00 & 405 & & & & & & & & & \\
\hline 119 & 1.00 & 402 & & 403 & & & & 402 & 402 & & 256 \\
\hline 120 & 1.00 & & 250 & & 337 & 340 & 335 & & & 337 & \\
\hline 121 & 1.00 & 248 & & & & & 248 & 337 & & 248 & 250 \\
\hline 122 & 1.00 & 266 & & 265 & 346 & 346 & & & 265 & 267 & 346 \\
\hline 123 & 1.00 & & 286 & 416 & & & 249 & 285 & & & 285 \\
\hline 124 & 1.00 & 262 & 264 & 265 & 263 & 260 & & & 263 & 265 & \\
\hline 125 & 1.00 & & 288 & & 287 & & 249 & & & 288 & 287 \\
\hline 126 & 1.00 & & 269 & & & & & 266 & & & \\
\hline 127 & 1.00 & 282 & & & & & & & & & 282 \\
\hline 128 & 1.00 & & & 266 & & & & & & & \\
\hline 129 & 1.00 & & 279 & 354 & & & & & & & \\
\hline 130 & 1.00 & & & 288 & & 288 & & & & & \\
\hline 131 & 1.00 & & & 269 & & & & & & & \\
\hline 132 & 1.00 & & 282 & & & & & & & & \\
\hline & 1.00 & 262 & & & & & & & & & \\
\hline 134 & 1.00 & & & & 267 & & & & & & \\
\hline$n_{i}$ & & 21 & 23 & 29 & 20 & 24 & 20 & 27 & 23 & 19 & 21 \\
\hline
\end{tabular}

a Interpret as animal 1, caught in trap 168, on day six.

has the effect of eliminating extremes and may be considered if one can assume the population to be stable during the sampling period. One might logically assume that if the population is stable during the trapping period, the best estimate would be that provided by the most trapping data - the last day with an estimate. This is expected since it includes the most complete information concerning the probability of trap avoidance, death, and possibly leaving the grid. Consequently, if one standard day were selected, it would logically be the estimate for the ninth day.

$$
p\left[\frac{47}{.44+1.96 \sqrt{\frac{(.44)(1-.44)}{1}}}\right]<47<\left[\frac{47}{.44-1.96 \sqrt{\frac{(.44)(1-.44)}{1}}}\right]=.95
$$

Eighteen simulation experiments were conducted, while holding the size constant and varying the size of home ranges and density, to further test the estimator's accuracy (Table 4). As far as the most reliable 
estimate over the nine day period is concerned, estimates for all days departed significantly from the known population size except those for day nine. However, if experiment 15 were deleted from the analyses, estimates for days eight and nine both failed to depart significantly from the actual population size. If the population were stable, it is suggested that the mean and median might also be considered effective estimates.

Table 2

$A_{i j}$ matrix for the data provided in Table 1, along with the essential parameters for using the estimator (EM-2).

\begin{tabular}{|c|c|c|c|c|c|c|c|c|c|c|c|c|c|}
\hline \multirow{2}{*}{$\begin{array}{l}\text { Trapping } \\
\text { day } \\
\text { (i) }\end{array}$} & \multirow{2}{*}{$n_{i}$} & \multirow{2}{*}{$m_{i}$} & \multirow{2}{*}{$\widehat{M}_{i}$} & \multicolumn{10}{|c|}{$A_{i j}$ Matrix } \\
\hline & & & & 1 & 2 & 3 & 4 & 5 & 6 & 7 & 8 & 9 & 10 \\
\hline 1 & 21 & 0 & 21 & & 10 & 7 & 1 & 1 & 0 & 2 & 0 & 0 & 0 \\
\hline 2 & 23 & 10 & 34 & & & 12 & 4 & 5 & 1 & 1 & 0 & 0 & 0 \\
\hline 3 & 29 & 19 & 44 & & & 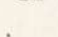 & 9 & 8 & 4 & 6 & 1 & 1 & 0 \\
\hline 4 & 20 & 14 & 50 & & & & & 8 & 4 & 4 & 2 & 2 & 0 \\
\hline 5 & 24 & 22 & 52 & & & & & & 8 & 6 & 2 & 4 & 1 \\
\hline 6 & 20 & 17 & 54 & & & & & & & 8 & 6 & 1 & 2 \\
\hline 7 & 27 & 27 & 52 & & & & & & & & 10 & 8 & 4 \\
\hline 8 & 23 & 21 & 51 & & & & & & & & & 3 & 5 \\
\hline 9 & 19 & 19 & 47 & & & & & & & & & & 9 \\
\hline 10 & 21 & 21 & 45 & & & & & & & & & & \\
\hline
\end{tabular}

Table 3

Parameters used in computing $E M-2$ as an estimator of small mammals.

\begin{tabular}{|c|c|c|c|c|c|c|c|c|c|c|c|}
\hline \multirow[b]{2}{*}{$\begin{array}{c}\text { Trapping } \\
\text { day }\end{array}$} & \multicolumn{4}{|c|}{ Probabilities } & \multicolumn{7}{|c|}{ Population estimates } \\
\hline & $\hat{P a}_{i}$ & $\hat{P} d_{i}$ & $\hat{P l_{i}}$ & $\hat{\Phi}_{i}$ & $d_{i}$ & $k_{i}$ & $l_{i}$ & $g_{i}$ & $\hat{M} D_{i}$ & $E M-2^{a}$ & $\mathrm{~N}_{i}$ \\
\hline 1 & .00 & 1.00 & .00 & .48 & .00 & 0 & .00 & 0 & .00 & 48 & 49 \\
\hline 2 & .00 & 1.00 & .00 & .56 & .00 & 0 & .00 & 0 & .00 & 52 & 49 \\
\hline 3 & .00 & 1.00 & .00 & .32 & .00 & 0 & .00 & 0 & .00 & 63 & 49 \\
\hline 4 & .03 & 1.00 & .00 & .44 & .00 & 0 & .00 & 0 & .00 & 55 & 49 \\
\hline 5 & .04 & .97 & .00 & .33 & 2.77 & 0 & .00 & 3 & .14 & 61 & 49 \\
\hline 6 & .11 & .92 & .00 & .50 & 2.44 & 0 & .00 & 3 & 1.32 & 54 & 49 \\
\hline 7 & .13 & .81 & .00 & .40 & 3.43 & 0 & .00 & 5 & 3.13 & 57 & 49 \\
\hline 8 & .26 & .69 & .00 & .37 & 6.03 & 0 & .00 & 14 & 5.71 & 51 & 49 \\
\hline 9 & .38 & .43 & .00 & .44 & .51 & 0 & .00 & 10 & 9.52 & 47 & 49 \\
\hline 10 & - & .05 & .00 & .00 & .00 & 0 & .00 & - & 12.08 & - & - \\
\hline
\end{tabular}

a Confidence limits on the ninth day estimate at the .95 level are $p(33<47<88)$.

The Chi-square test resulted in totals of 32.59 and 32.90 for the means and medians respectively; both being slightly larger than $\chi^{2}$ for day eight (31.0), but appreciably greater than $\chi^{2}$ for day nine (25.2).

Although one wouldn't expect the $\chi^{2}$ values for any given experiment to be particularly low they can be used to determine which data sets result in extreme departures from known population size. The two 
extreme departures in the 18 simulated experiments are no. 5 and 15. Although others are relatively high, reasonable estimates are usually available from one of the methods mentioned previously. There seems to be no reason for these infrequent wide departures, and they are most likely simply a matter of chance, and are rather difficult to identify unless the daily estimates are extremely erratic as is the case with expt. no. 5 .

Table 4

Population estimates $(E M-2)$ for 18 simulation experiments, along with $\chi^{2} /$ analyses of the results, for stable population.

\begin{tabular}{|c|c|c|c|c|c|c|c|c|c|c|c|c|c|}
\hline \multirow{2}{*}{$\begin{array}{l}\text { Simula- } \\
\text { tion expt. } \\
\text { No. }\end{array}$} & \multirow{2}{*}{$\begin{array}{l}\text { Ac- } \\
\text { tual } \\
\text { pop. } \\
\text { size }\end{array}$} & \multicolumn{9}{|c|}{$\begin{array}{c}\text { Population estimates for each trap- } \\
\text { ping day (i)a }\end{array}$} & \multirow{2}{*}{$\underset{(\bar{x})}{\operatorname{Mean}}$} & \multirow{2}{*}{$\begin{array}{l}\text { Home } \\
\text { range } \\
\text { radius }^{b} \\
(\mathrm{~m})\end{array}$} & \multirow{2}{*}{$\begin{array}{l}\text { Chi } \\
\text { squ- } \\
\text { are }\end{array}$} \\
\hline & & 1 & 2 & 3 & 4 & 5 & 6 & 7 & 8 & 9 & & & \\
\hline 1 & 18 & 12 & 24 & $12 \mathrm{c}$ & 15 & 32 & 12 & 12 & 11 & 21 & 16.7 & 30 & $24.6^{*}$ \\
\hline 2 & 18 & 6 & 5 & 10 & 17 & 49 & $16 \mathrm{c}$ & 17 & 16 & 15 & 16.7 & 30 & $75.5^{*}$ \\
\hline 3 & 30 & 21 & 66 & $28 \mathrm{c}$ & 42 & 28 & 24 & 34 & 28 & 33 & 33.7 & 30 & $53.0^{*}$ \\
\hline 4 & 30 & 20 & 41 & 34 & 25 & $28 \mathrm{c}$ & 35 & 31 & 22 & 27 & 29.2 & 30 & 11.8 \\
\hline 5 & 36 & 65 & $61 c_{1}$ & 125 & 87 & 68 & 32 & 31 & 36 & 40 & 60.5 & 30 & $362.9 *$ \\
\hline 6 & 36 & 59 & 27 & $37 c$ & 53 & 28 & 60 & 21 & 37 & 30 & 39.1 & 30 & $50.1^{*}$ \\
\hline 7 & 17 & 14 & 16 & 21 & 14 & 18 & 16 & 25 & $17 c$ & 18 & 17.7 & 44 & 6.1 \\
\hline 8 & 17 & 20 & 19 & 15 & 17 & 16 & 19 & 20 & $18 \mathrm{c}$ & 17 & 17.8 & 44 & 1.8 \\
\hline 9 & 35 & 57 & 40 & 42 & 42 & 37 & $38 \mathrm{c}$ & 36 & 34 & 32 & 39.7 & 44 & $18.0^{*}$ \\
\hline 10 & 35 & 44 & 38 & 33 & 62 & 41 & $35 c$ & 32 & 33 & 29 & 38.6 & 44 & $25.9 *$ \\
\hline 11 & 49 & 41 & 48 & 68 & 60 & 59 & $53 c$ & 55 & 48 & 49 & 53.4 & 44 & $14.2^{*}$ \\
\hline 12 & 49 & 48 & 52 & 63 & 55 & 61 & $54 c$ & 57 & 51 & 47 & 54.2 & 44 & $9.8^{*}$ \\
\hline 13 & 13 & 19 & 24 & 18 & 15 & 15 & 15 & 20 & $17 \mathrm{c}$ & 16 & 17.6 & 54 & $20.6^{*}$ \\
\hline 14 & 13 & 10 & 32 & 18 & 21 & $19 \mathrm{c}$ & 19 & 19 & 18 & 18 & 19.3 & 54 & $47.5^{*}$ \\
\hline 15 & 16 & 30 & 35 & $32 c$ & 31 & 35 & 33 & 34 & 32 & 31 & 32.6 & 54 & $156.1^{*}$ \\
\hline 16 & 16 & 37 & $26 c$ & 21 & 29 & 27 & 27 & 25 & 24 & 23 & 26.6 & 54 & $73.5^{*}$ \\
\hline 17 & 43 & 64 & 51 & 59 & 61 & $55 c$ & 57 & 54 & 52 & 49 & 55.8 & 54 & $38.7^{*}$ \\
\hline 18 & 43 & 44 & $49 c$ & 47 & 53 & 59 & 57 & 52 & 48 & 45 & 50.4 & 54 & $16.7^{*}$ \\
\hline \multirow{2}{*}{\multicolumn{2}{|c|}{ Chi Square }} & \multicolumn{9}{|c|}{ * $31.0 *$} & & & \\
\hline & & 126.2 & & $268.0^{*}$ & & $143.5^{*}$ & & $52.9^{*}$ & & $25.2^{*}$ & & & \\
\hline
\end{tabular}

a Estimates, using $E M-2$ are not obtainable on the last day of a trapping period; thus, estimates are not listed for $i=10$.

b Home range radii were computed according to the methods described by $\mathrm{B} u \mathrm{rg}$ \& J orgensen (1973).

c Median.

* Significant difference at the .95 level.

Natural populations probably do not usually remain stable during the trapping period, although change in the members may be rather subtle in either a positive or negative direction. Also, birth and death might compensate to the extent that the actual population fluctuates very little, if any. These presumed changes during the trapping periods were imposed on different populations at varying rates (Table 5). If one assumes the population size is changing, or even if the composition is 


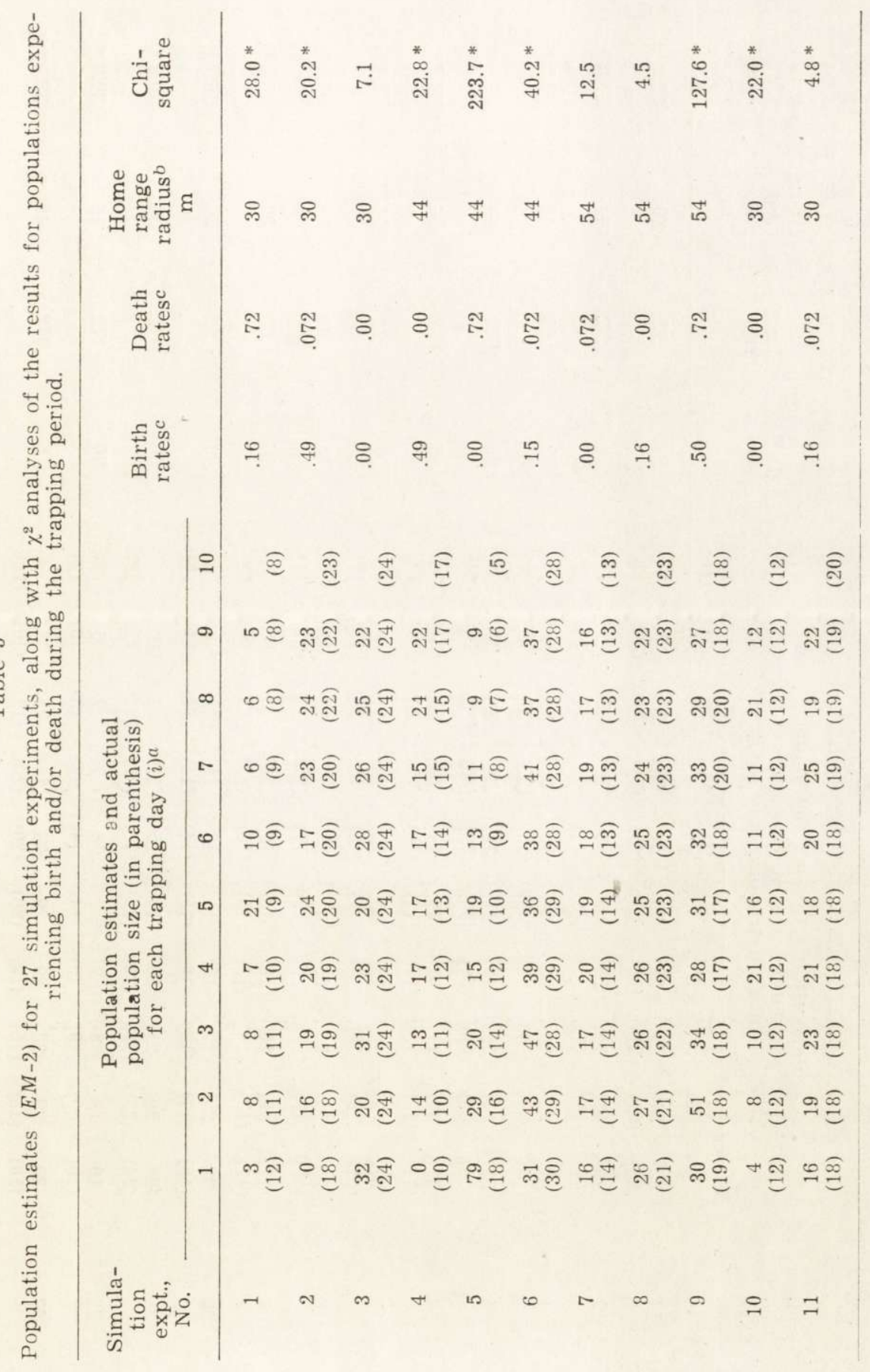




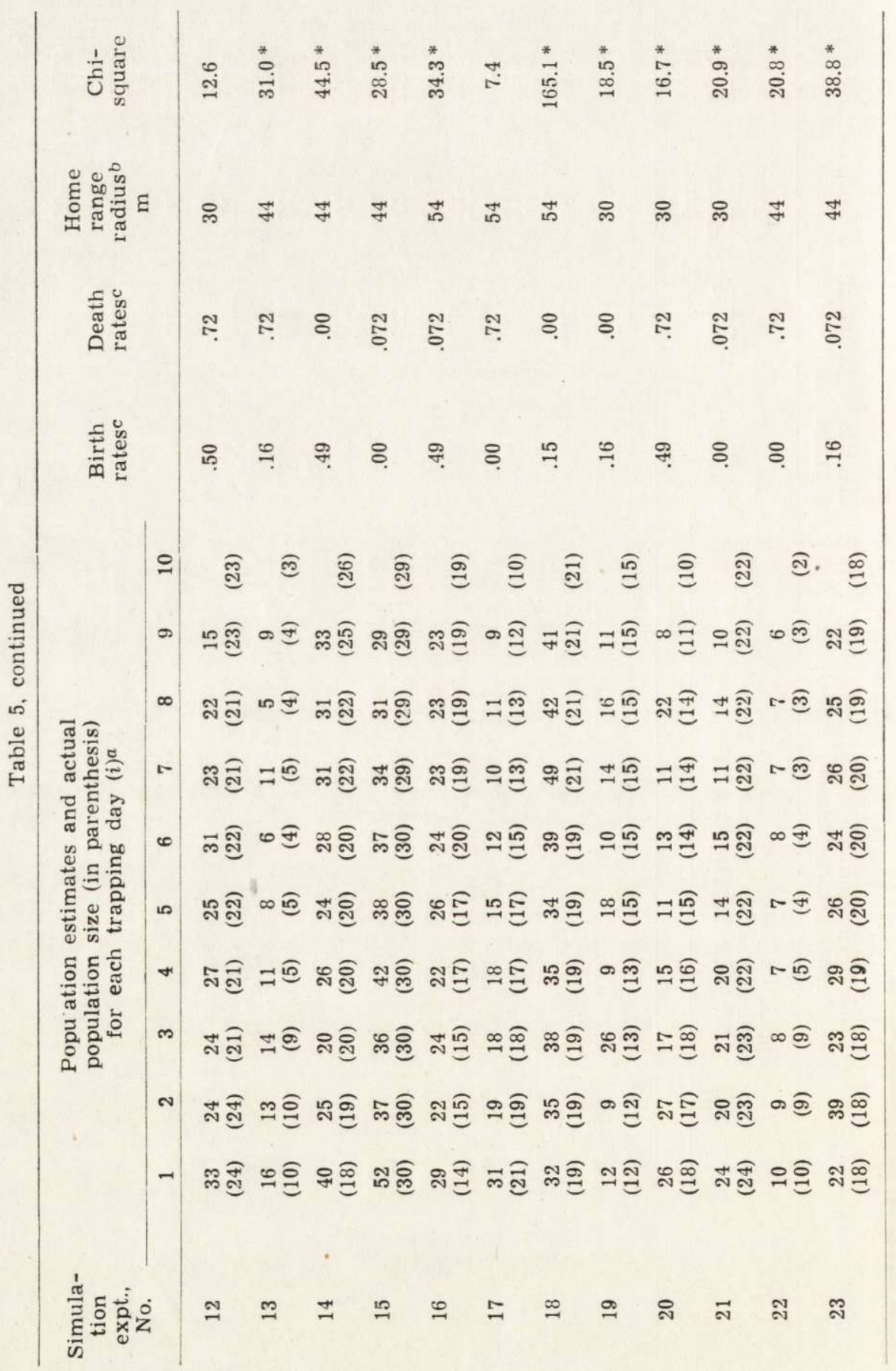




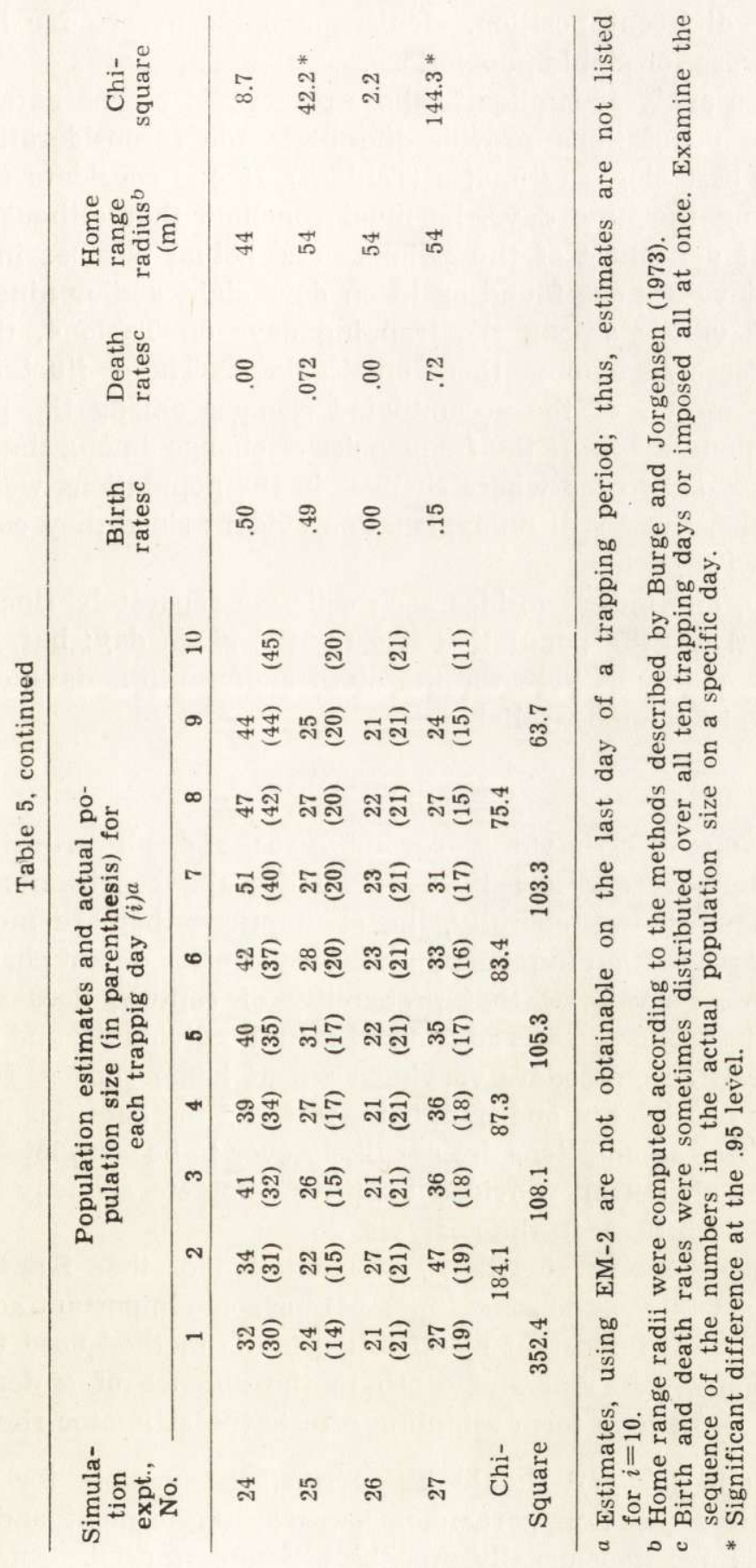


changing with no change in size, the estimates must be considered from an entirely different position. Medians and means are far less logical than they were for stable populations.

Chi square analyses similar to those performed on the data generated from stable populations provide the basis for reconsideration of the criteria for best single estimates (Table 5). If one considers only the $\chi^{2}$ values among the nine days, he must conclude that although there is a significant departure of the estimate and actual number in all cases, the smallest values are found again on days eight and/or nine.

Unlike $\chi^{2}$ values among the trapping days on Table 4 , there is no continual decrease among them on Table 5. These fluctuations are obviously a matter of the accumulated changes among the populations due to birth and death. If the frequencies of change among the 27 experiments are totaled to see where changes in the populations were actually occurring, there was still no explanation evident since they could not be correlated with $\chi^{2}$ changes.

Generally, one must consider each estimate separately since they are keyed directly to the population size for the given day; but, if a single estimate is required, those for the eigth and/or ninth days, of trapping are likely to be the most reliable.

\section{DISCUSSION}

Small mammal estimators are numerous and all are designed to estimate numbers and densities, but under the constraints of rather specific assumptions. Generally, the assumptions that are most binding are: (1) that population numbers and activity centers do not change during the sampling period; (2) that probability of capture does not change during the trapping period and is equal for all individuals; and (3) that the estimator can accommodate varying sizes of home ranges, from which data can be obtained in one trapping effort and the densities determined. Although these assumptions are not likely ever to be completely satisfied, S mith, et al. (1972) provided a rather complete strategy and model for dealing with them in the analyses.

This paper demonstrates the implementation of the $\mathrm{Smith}$ et al. (1972) model while at the same time, making some important adjustments. Perhaps the most important modification is the method used to partition trap avoidance, dispersal and death in the absence of a dense line of traps (Fig. 1). In long term sampling efforts the estimator should approximate $N$ and $(\hat{N}-N)^{2}$ should be as small as possible for best accuracy. But, if population parameters such as recruitment and mortality are unknown, as they usually are, the estimator must be robust enough 
to accommodate the changes; and if possible, provide some indication of what is actually happening to the population during the trapping period. The estimator $(E M-2)$ described and demonstrated in this paper, attempts to deal with these problems.

The field design seems quite adequate to provide the necessary data and performed precisely as S mith et al. (1972) predicted it would, except the dense-line tended to prevent dispersal rather than measuring it. This was possibly due to its placement. Few animals in all of the experiments were recorded as coming onto or leaving the grid, possibly because of the distance between the grid and the dense-line. Indications are that this distance can be reduced and provide even better data from which the parameters can be partitioned, along with a meaningful reduction in the field effort. The experiments reported here are essentially without dense-line data, since so few animals used it. Better estimates of $N_{i}$ would have been obtained if the dense-line did provide data that would assist in the partitioning.

Efficiencies of this estimator (EM-2) are observed in Tables 4 and 5 , where considerable departures are evident. This is particularly true when data were obtained from a population that deliberately failed to satisfy the assumptions stated earlier (Table 5). Perhaps the most important consideration at this point is whether or not the estimator tries to adjust to changes in the population; and in most cases, it does although not as rapidly as would be desired. Whether or not these departures are greater or less than what other methods provide must be analysed further, since EM-2 might still provide the most accurate estimate. A comparative study is presently being conducted and the results will be available soon.

A most serious limitation of many estimators is the lack of meaningful methods for obtaining confidence limits on the estimate. The $E M-2$ is no exception, although the method described is acceptable. Its basic problems are inevitable wide limits, in most cases too wide to be helpful unless there are rather large numbers of animals moving onto the grid. If the methods for estimating confidence are good, the results would suggest that the dense-line could be considerably closer to the grid, perhaps even the outer two lines of traps on the grid.

\section{REFERENCES}

1. Burge J. R. \& Jorgensen C. D., 1973: Home range of small mammals: a reliable estimate. J. Mammal. 54: 483-488.

2. Hayne D. W., 1949: Two methods for estimating population from trapping records. J. Mammal. 30: 399-411.

3. Jol1y G. M., 1963: Estimates of population parameters from multiple recapture data with death and dilution-deterministic model. Biometrika 50: 113-123. 
4. Jolly G. M., 1965: Explicit estimates from capture-recapture data with both death and immigration-stochastic model. Biometrika 52: 225-247.

5. Jorgensen C. D., Scott D. T. \& Smith H. D., 1972: Small-mammal trapping simulator. Proc. Sum. Comp. Sim. Conf., 2: 1154-1168.

6. Jorgensen C. D., Smith H. D. \& Scott D. T., 1972: Evaluation of techniques for estimating population sizes of desert rodents. US-IBP Desert Biome Rpt. RM72-26: 1-196.

7. Leslie P. H., 1952: The estimation of population parameters obtained by means of capture-recapture method II: the estimation of total numbers. Biometrika 39: $363-388$.

8. Leslie P. H. \& Chitty D., 1951: The estimation of population parameters from data obtained by means of the capture-recapture model I: the maximum likelihood equations for estimating the death rates. Biometrika 38: 269-292.

9. Lincoln F. C., 1930: Calculating water fowl abundance on the basis of banding returns. U. S. Dept. Agric. Cir. 118: 1-4.

10. Petersen C. G. J., 1896: The yearly immigration of young plaice in the Limjord from the German Sea. Rpt. Danish Biol. Stat. 6: 1-48.

11. Schnabel Z. E., 1938: The estimation of the total fish population of a lake. Am. Math. Monthly 45: 384-353.

12. Smith H. D., Jorgensen C. D. \& Tolley H. D., 1972: Estimation of small mammal using recapture methods: partitioning of estimator variables. Acta theriol. 17: 57-66.

Accepted, November 6, 1974.

Department of Zoology,

Brigham Young University,

Provo, Utah 84602, U.S.A.

Clive D. JORGENSEN, H. Duane SMITH i Del T. SCOTT

OCENA LICZEBNOSCI MAEYCH SSAKOW METODA POWTÓRNYCH ZEOWIEN, ZE ZMIENNYMI DZIELONYMI

\section{Streszczenie}

Metoda oznaczania liczebności i zagęszczenia drobnych ssaków z podziałem estymatora została przedstawiona przez Smitha, Jorgensena i Tolley'a w 1972 roku. Choć ich model $(E M-1)$ nie był zbyt praktyczny, bowiem wymagał danych uzyskiwanych na powierzchniach o dużej gęstości linii, to jednak stworzył podstawę do niniejszych modyfikacji i metod uzupełniających. Główna zmiana polega na podaniu estymatora $(E M-2)$, który nie wymaga dużego zagęszczenia linii ani dużego zagęszczenia pułapek. Obecna metoda może być używana prawie do wszystkich rodzajów powierzchni odłownych, o ile stosuje się łowienie i wypuszczanie zwierząt.

Podana metoda pozwala także na ustalenie danych dla poszczególnych dni całego okresu odłowów a więc uchwycenie zmian w badanej populacji. Większość estymatorów cechuje się zbyt małą liczbą potrzebnych wskaźników, natomiast EM-2 posiada wiele wskaźników przeliczeniowych i współczynników prawdopodobieństwa, które są pomocne przy obliczaniu parametrów populacyjnych.

Dla przykładu pokazano jak działa przyjęty estymator $w$ odniesieniu do symulowanych populacji, w sytuacji kiedy liczebność była stała. Stanowiło to podstawę do oceny wyników innych badań. Ogólnie można powiedzieć, że ustalenia było najwierniejsze dla ostatnich dni odłowów, nawet w sytuacji kiedy liczebność populacji była zmienna. 